\title{
The role of Src kinase in the biology and pathogenesis of Acanthamoeba castellanii
}

\author{
Ruqaiyyah Siddiqui ${ }^{1}$, Junaid lqbal ${ }^{1}$, Marie-josée Maugueret ${ }^{2}$ and Naveed Ahmed Khan ${ }^{1 *}$
}

\begin{abstract}
Background: Acanthamoeba species are the causative agents of fatal granulomatous encephalitis in humans. Haematogenous spread is thought to be a primary step, followed by blood-brain barrier penetration, in the transmission of Acanthmaoeba into the central nervous system, but the associated molecular mechanisms remain unclear. Here, we evaluated the role of Src, a non-receptor protein tyrosine kinase in the biology and pathogenesis of Acanthamoeba.
\end{abstract}

Methods: Amoebistatic and amoebicidal assays were performed by incubating amoeba in the presence of Src kinase-selective inhibitor, PP2 (4-amino-5-(4-chlorophenyl)-7-(t-butyl)pyrazolo[3,4- $d$ ]pyrimidine) and its inactive analog, PP3 (4-amino-7-phenylpyrazolo[3,4-d]pyrimidine). Using this inhibitor, the role of Src kinase in A. castellanii interactions with Escherichia coli was determined. Zymographic assays were performed to study effects of Src kinase on extracellular proteolytic activities of A. castellanii. The human brain microvascular endothelial cells were used to determine the effects of Src kinase on A. castellanii adhesion to and cytotoxicity of host cells.

Results: Inhibition of Src kinase using a specific inhibitor, PP2 (4-amino-5-(4 chlorophenyl)-7-(t-butyl)pyrazolo [3,4- $d$ ] pyrimidine) but not its inactive analog, PP3 (4-amino-7-phenylpyrazolo[3,4-d] pyrimidine), had detrimental effects on the growth of A. castellanii (keratitis isolate, belonging to the T4 genotype). Interestingly, inhibition of Src kinase hampered the phagocytic ability of A. castellanii, as measured by the uptake of non-invasive bacteria, but, on the contrary, invasion by pathogenic bacteria was enhanced. Zymographic assays revealed that inhibition of Src kinases reduced extracellular protease activities of $A$. castellanii. Src kinase inhibition had no significant effect on $A$. castellanii binding to and cytotoxicity of primary human brain microvascular endothelial cells, which constitute the blood-brain barrier.

Conclusions: For the first time, these findings demonstrated that Src kinase is involved in A. castellanii proliferation, protease secretions and phagocytic properties. Conversely, invasion of Acanthamoeba by pathogenic bacteria was stimulated by Src kinase inhibition.

Keywords: Acanthamoeba, Pathogenesis, Encephalitis, Src kinase

\section{Background}

Based on the ribosomal DNA (rDNA) sequencing, the genus Acanthamoeba represents 17 different groups, i.e., T1 - T17 [1-3]. The basis of this scheme is that each group (or genotype) exhibits $\geq 5 \%$ rDNA sequence divergence from other genotypes. Pathogenic Acanthamoeba (predominantly belonging to the T4 genotype) can produce painful, blinding keratitis, normally associated with contact lens use or a fatal granulomatous amoebic

\footnotetext{
* Correspondence: naveed5438@gmail.com

${ }^{1}$ Department of Biological and Biomedical Sciences, The Aga Khan University, Karachi, Pakistan

Full list of author information is available at the end of the article
}

encephalitis (GAE), primarily associated with immunocompromised patients [4-6]. The most distressing aspect is that the prognosis is poor, despite advances in antimicrobial chemotherapy and supportive care. In particular, there is very limited success in the treatment of GAE, which is most likely due to the inability of drugs to cross the blood-brain barrier into the central nervous system (CNS) to target pathogen, non-specific toxicity, and amoebae transformation into resistant cyst forms. However, alkylphosphocholine compounds show promise [7]. Among them, hexadecylphosphocholine has been shown to possess anti-Acanthamoeba characteristics and

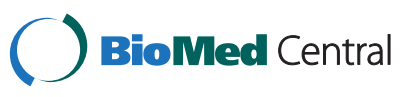


has the ability to cross the blood-brain barrier. To date, their mode of action and in vivo efficacy are unknown. Clearly, there is a need to find novel strategies in the rational development of therapeutic interventions.

The burden of Acanthamoeba keratitis on human health is estimated at $0.01-1.5$ infections per 10,000 people who wear contact lens [4]. In contrast, a true or even approximate burden of encephalitis on human health is not known. As indicated above, GAE infections are usually limited to immunocompromised patients, such as those with HIV/AIDS [8]. GAE can also occur in chronically ill or debilitated individuals, some of whom take immunosuppressive therapy or broad-spectrum antibiotics [8]. The pathogenesis of the disease is not clearly understood, although the route of infection is thought to relate to the inhalation of the amoebae through the nasal passages and lungs or infection through skin lesions [8]. The respiratory and cutaneous infections tend to last for a few months, whereas infection involving the CNS can be fatal within days $[5,6]$. The haematogenous spread by circulating amoebae is a prerequisite for GAE, followed by their traversal of the blood-brain barrier, but the underlying mechanisms remain incompletely understood $[4,9]$. It is possible that new targets may be found in the signal transduction pathways that can affect amoeba survival and hostpathogen interactions. Src is a member of a larger family of related tyrosine kinases that includes Fyn, Yes, Lck, Blk, Lyn, Hck, Yrk and Fgr. Src is a non-receptor protein tyrosine kinase and its activation is mainly regulated by phosphorylation at the tyrosine 416 residue [10]. Src signalling has been implicated in a variety of cellular processes, including cell growth, survival, cellular transformation and motility [11,12]. For the first time, in the present study, we investigated the role of Src kinase in A. castellanii.

\section{Methods}

All chemicals were purchased from Sigma (Poole, Dorset, UK), unless otherwise stated.

\section{Culturing of Acanthamoeba castellanii}

An A. castellanii isolate belonging to the T4 genotype was obtained from the American Type Culture Collection (ATCC50492), and sourced from a keratitis patient. The cells were grown axenically in $10 \mathrm{ml}$ of PYG medium $[0.75 \%(\mathrm{w} / \mathrm{v})$ proteose peptone, $0.75 \%(\mathrm{w} / \mathrm{v})$ yeast extract and $1.5 \%(\mathrm{w} / \mathrm{v})$ glucose] (Oxoid Ltd., Basingstoke, UK) in a T-75 tissue culture flask at $30{ }^{\circ} \mathrm{C}$ as previously described [13]. The medium was refreshed $17-20 \mathrm{~h}$ prior to experiments, which resulted in $>95 \%$ of amoebae in the trophozoite form.

\section{Human brain microvascular endothelial cell (HBMEC) culture}

The primary BMEC were isolated from the human tissue and purified by fluorescent activated cell sorting (FACS) and exhibited endothelial characteristics, such as expression of endothelial markers, F-VIII, carbonic anhydrase IV and uptake of acetylated low density lipoprotein (AcLDL) as previously described [13,14]. HBMEC were grown in RPMI-1640 containing 10\% foetal bovine serum, 10\% NuSerum, $2 \mathrm{mM}$ glutamine, $1 \mathrm{mM}$ pyruvate, penicillin $(100 \mathrm{U} / \mathrm{ml})$, streptomycin $(100 \mathrm{U} / \mathrm{ml})$, nonessential amino acids and vitamins (Invitrogen, Paisley, UK) $[13,14]$.

\section{Amoebistatic and amoebicidal assays}

A. castellanii were grown to confluency in 24-well plates. Next day, plates were washed with PBS to remove unbound amoebae. The varying concentrations of a potent, Src kinase-selective inhibitor, PP2 (4-amino-5-(4chlorophenyl)-7-( $t$-butyl)pyrazolo[3,4- $d]$ pyrimidine) [15] and its inactive analog, PP3 (4-amino-7-phenylpyrazolo [3,4-d]pyrimidine) [16] (Calbiochem, San Diego, CA, USA) were added. For growth assays, amoebae plus inhibitors were incubated in PYG medium for various intervals of time, followed by haemocytometer counting. For viability assays, amoeba plus inhibitors were incubated in PBS for $24 \mathrm{~h}$, and viability determined by Trypan blue exclusion testing using haemocytometer counting [17]. For controls, normal growth rates of $A$. castellanii were determined using growth medium alone, i.e., PYG or amoebae incubated in PBS in the absence of inhibitors.

\section{Zymographic assays}

The extracellular proteolytic activities of Acanthamoeba were determined using zymographic assays as previously described [18]. Briefly, A. castellanii were incubated in the presence or absence of various concentrations of Src kinase inhibitors for $24 \mathrm{~h}$. Next day, cell-free supernatants (CM, conditioned medium) were collected by centrifugation. The CM were electrophoresed on sodium dodecyl sulfate-polyacrylamide gel electrophoresis (SDS-PAGE) containing gelatin $(2 \mathrm{mg} / \mathrm{ml})$ as a protease substrate. After electrophoresis, gels were washed in $2.5 \%$ Triton X-100 (wt/vol) for $60 \mathrm{~min}$, then incubated in developing buffer

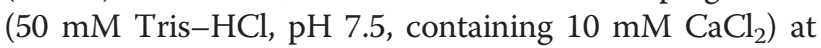
$37^{\circ} \mathrm{C}$ overnight. Next day, gels were stained with Coomassie Brilliant Blue. Areas of gelatin digestion were visualised as non-staining regions in the gel.

\section{Phagocytosis assays using live, non-invasive $E$. coli $\mathrm{K}-12$}

A. castellanii were grown to confluency in 24-well plates. Next day, plates were washed with PBS to remove unbound amoebae. A. castellanii were incubated with various concentrations of Src kinase inhibitor, PP2 and 
its inactive analog, PP3 in RPMI at room temperature for $30 \mathrm{~min}$. After this incubation, A. castellanii were washed with PBS to remove any residual inhibitor. Next, live Escherichia coli $\mathrm{K}-12$ (10 $/$ well), a non-invasive laboratory strain, HB101 were added and plates were incubated for $45 \mathrm{~min}$ to allow phagocytic uptake. Following this incubation, supernatants were removed and gentamicin was added (final conc. $100 \mu \mathrm{g} / \mathrm{ml}$ for $45 \mathrm{~min}$ ) to kill any remaining extracellular $E$. coli. Next, the numbers of $A$. castellanii were determined using haemocytometer counting. Finally, A. castellanii were solubilized with $0.5 \%$ SDS and E. coli counts were determined by inoculating lysates on nutrient agar plates. This allowed the determination of any intracellular $E$. coli.

\section{Determination of relative phagocytic activity}

The level of $A$. castellanii phagocytosis was determined as follows: No. of $E$. coli colony forming units (cfu)/Total number of $A$. castellanii x $100=\%$ phagocytosis. Results are expressed as relative phagocytosis (\% phagocytosis in untreated A. castellanii was considered as $100 \%$ and levels of phagocytosis in inhibitor-treated A. castellanii are shown as percentage change).

\section{Invasion assays using live pathogenic E. coli K1}

Invasion assays were performed as for phagocytosis assays except that an invasive strain of $E$. coli K1, RS218 (O18:K1: H7) a cerebrospinal fluid isolate from a meningitis patient) was used as opposed to the non-invasive K-12.

\section{Adhesion assays}

To determine the ability of $A$. castellanii to bind HBMEC, adhesion assays were performed [19]. Briefly, HBMEC were grown to confluency in 24-well plates. $A$. castellanii (4 x $10^{5}$ amoebae/well) were pre-incubated with various concentrations of inhibitor for $30 \mathrm{~min}$. After this incubation, A. castellanii were washed with PBS to remove residual inhibitor. Finally, A. castellanii were added to HBMEC monolayers in RPMI 1640 containing $2 \mathrm{mM}$ glutamine, $1 \mathrm{mM}$ pyruvate and nonessential amino acids) and plates incubated at $37^{\circ} \mathrm{C}$ in a $5 \% \mathrm{CO}_{2}$ incubator. After $1 \mathrm{~h}$ incubation, the unbound amoebae were counted using a haemocytometer and the numbers of bound amoebae were calculated as follows: No. of unbound amoebae/Total number of amoebae $\mathrm{x}$ $100=\%$ unbound amoebae. The numbers of bound amoebae were deduced as follows: \% unbound amoebae $-100=\%$ bound amoebae.

\section{Cytotoxicity assays}

Cytotoxicity assays were performed as previously described [19]. Briefly, A. castellanii were pre-treated with PP2 and PP3 and incubated with HBMEC monolayers grown in 24-well plates as described for adhesion assays.
Plates were incubated at $37{ }^{\circ} \mathrm{C}$ in a $5 \% \mathrm{CO}_{2}$ incubator and periodically observed for cytopathic effects for up to $24 \mathrm{~h}$. At the end of this incubation period, supernatants were collected and cytotoxicity was determined by measuring lactate dehydrogenase (LDH) release (cytotoxicity detection kit; Roche Applied Science, Lewes, East Sussex, UK). Briefly, conditioned media of co-cultures of A. castellanii and HBMEC were collected and percentage LDH was detected as follows: (sample value - control value/total LDH release - control value $\mathrm{x} 100=\%$ cytotoxicity). Control values were obtained from HBMEC incubated in RPMI alone. Total LDH release was determined from HBMEC treated with $1 \%$ Triton X-100 for 30 min at $37^{\circ} \mathrm{C}$.

\section{Acanthamoeba Src kinase identification and sequence alignments}

Putative Acanthamoeba Src kinase protein was searched in Acanthamoeba castellanii genome database, hosted by The Human Genome Sequencing Center, Baylor College of Medicine (http://blast.hgsc.bcm.tmc.edu/blast. hgsc ?organism = AcastellaniNeff), using closely related Monosiga brevicollis Src1 kinase protein sequence (NCBI accession No. AAP78682) [20], through BLAST search. Recovered Acanthamoeba protein was further aligned with its different homologs in other organisms using MUSCLE 3.8 hosted at European Bioinformatics Institute webpage (http://www.ebi.ac.uk/Tools/msa/muscle/). The name and NCBI accession numbers of different organisms Src kinase proteins used in alignment, are Drosophila melanogaster c-Src (AAA28913.1), Caenorhabditis elegans Src1 (NP_490866), Ephydatia fluviatilis Src related protein (BAB83688), Monosiga brevicollis Src1 (EDR48627.1) Hydra vulgaris (AAA29217.1) and Human cSrc (NP_005408).

\section{Results and discussion}

\section{Inhibition of src kinase reduced A. castellanii growth}

To determine the role of Src kinase on the biological properties of $A$. castellanii, growth and viability assays were performed. Growth under control conditions (amoebae incubated in growth medium in the absence of inhibitor) showed an approximate doubling of the number of cells every $24 \mathrm{~h}$ (Figure 1). However, the presence of Src kinase inhibitor, PP2, significantly reduced growth $[P=0.015$ for amoebae in PYG versus amoebae in PYG plus PP2 $(50 \mu \mathrm{M})$ at $96 \mathrm{~h}$, using $T$-test, paired, one tail distribution] but did not affect amoebic viability, as determined by Trypan blue exclusion test ( $0 \%$ effects). At micromolar concentrations of PP2 $(50 \mu \mathrm{M})$, amoeba growth was abolished. In contrast, inactive analog Src kinase, PP3 affected neither growth nor viability of $A$. castellanii for any time point tested $[P=0.051$ for amoebae in PYG versus amoebae in PYG plus PP3 $(50 \mu \mathrm{M})$ at $96 \mathrm{~h}$, using $T$-test, paired, one tail distribution] (Figure 1). 


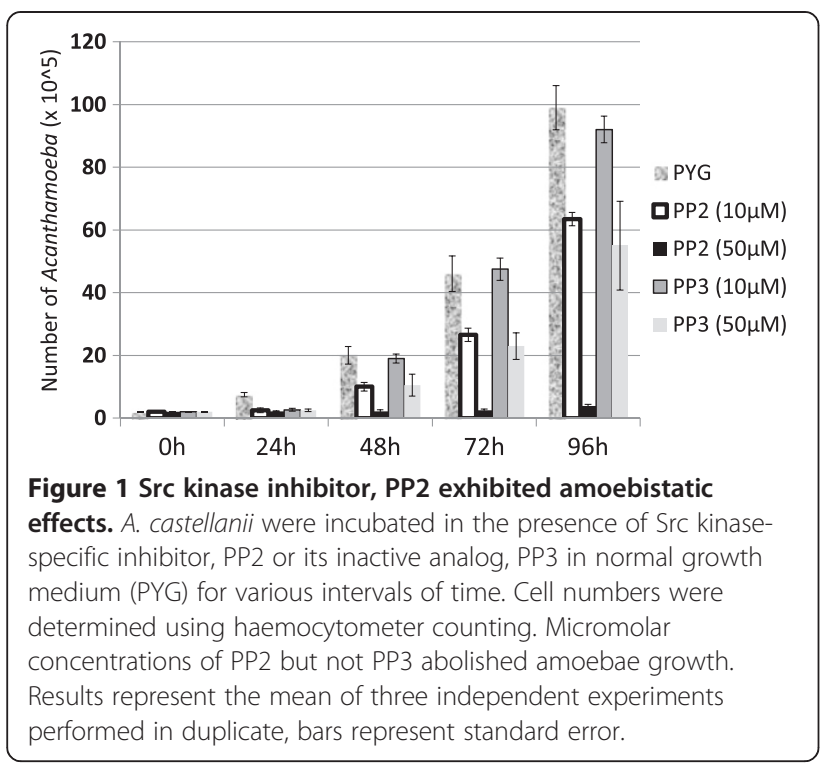

Src kinase inhibition blocked $A$. castellanii phagocytosis in a concentration-dependent manner

To determine the effects of Src kinases on A. castellanii phagocytosis of $E$. coli $\mathrm{K}-12$, phagocytosis assays were performed. It was observed that A. castellanii exhibited significant reduced bacterial uptake in the presence of PP2, specific Src kinase inhibitor $[P=0.038$ for amoebae with K-12 versus amoebae with K-12 plus PP2 $(50 \mu \mathrm{M})$, using $T$-test, paired, one tail distribution], although there was no difference at $10 \mu \mathrm{M}(P=0.21)$ (Figure 2). At 100 $\mu \mathrm{M}$ of PP2, more than $50 \%$ inhibition of $A$. castellanii uptake of E. coli K-12 strain, HB101 was observed (Figure 2). In contrast, PP3, an inactive analog of PP2, had no effect on bacterial uptake by $A$. castellanii at any of the concentrations tested $(P=0.127$ for amoebae with $\mathrm{K}-12$ versus amoebae with $\mathrm{K}-12$ plus PP3 $(50 \mu \mathrm{M})$, using $T$-test, paired, one tail distribution) (Figure 2).

\section{Pathogenic E. coli $\mathrm{K} 1$ invasion of A. castellanii was} enhanced using Src kinase inhibitor, PP2

In contrast to phagocytic uptake of non-invasive bacteria by Acanthamoeba (Figure 2), effects of Src kinase were reversed in the invasion of pathogenic E. coli $\mathrm{K} 1$ into $A$. castellanii. Pre-treatment of $A$. castellanii with PP2 significantly enhanced invasion of pathogenic bacteria $[P=0.026$ for amoebae with $\mathrm{K}-12$ versus amoebae with $\mathrm{K}-12$ plus PP2 $(50 \mu \mathrm{M})$, using $T$-test, paired, one tail distribution], although there was no difference at $10 \mu \mathrm{M}$ $(P=0.077)$ (Figure 3). At $100 \mu \mathrm{M}$ of $\mathrm{PP} 2$, invasion of a neuropathogenic E. coli $\mathrm{K} 1$ into A. castellanii was more than doubled (Figure 3). In contrast, PP3, an inactive analog of PP2 had no effect on bacterial uptake by $A$. castellanii at any of the concentrations tested $[P=0.09$ for amoebae with K-12 versus amoebae with $\mathrm{K}-12$ plus

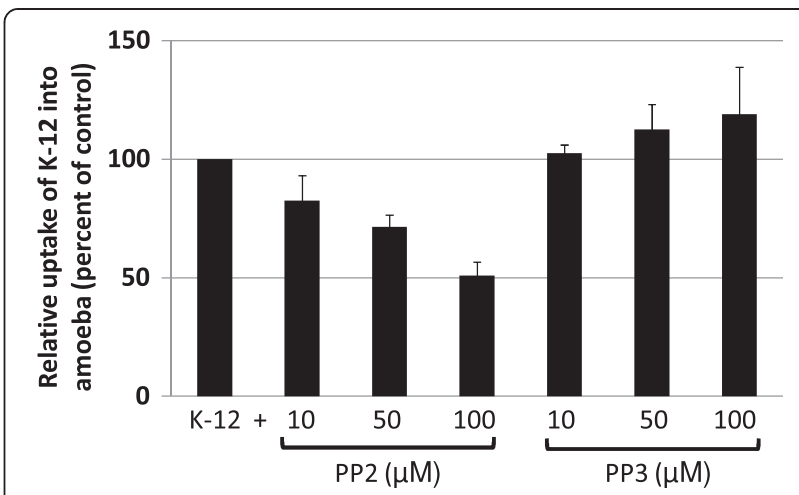

Figure 2 Inhibition of Src kinase blocks $A$. castellanii phagocytosis. The effects of Src kinase in the ability of $A$. castellanii to uptake live non-invasive E. coli strain, HB101. Untreated A. castellanii were used as $100 \%$ phagocytosis and levels of phagocytosis in inhibitor-treated A. castellanii are shown as percentage change. Note that Src kinase inhibitor, PP2 but not its inactive analog, PP3 exhibits a significant decrease in A. castellanii phagocytosis at $50 \mu \mathrm{M}(P<0.05)$. Results represent the mean of three independent experiments performed in duplicate, bars represent standard error.

PP3 $(50 \mu \mathrm{M})$, using $T$-test, paired, one tail distribution] (Figure 3).

\section{Src kinases are involved in Acanthamoeba protease secretions}

To determine the role of Src kinase in A. castellanii protease secretion, conditioned medium (CM) was produced in the presence of Src kinase inhibitor, PP2, or its inactive analog, PP3 and analysed for protease activities.

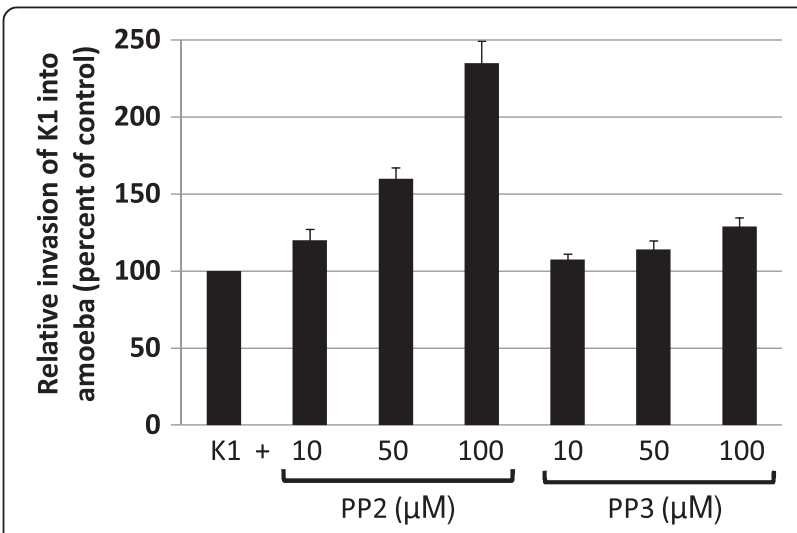

Figure 3 Src kinase inhibition enhanced neuropathogenic $E$. coli $\mathbf{K} 1$ invasion of $A$. castellanii. The involvement of Src kinase in the invasion of pathogenic $E$. coli (meningitis isolate) into $A$. castellanii was determined. Untreated $A$. castellanii were used as $100 \%$ phagocytosis and levels of bacterial invasion in inhibitortreated A. castellanii are shown as percentage change. Src kinase inhibitor, PP2 but not its inactive analog, PP3 exhibits a significant increase in bacterial invasion of $A$. castellanii $(P<0.05)$. Results represent the mean of three independent experiments performed in duplicate, bars represent standard error of the mean. 
In the absence of any inhibitor, A. castellanii exhibited significant proteolytic activities (Figure 4). The proteolytic activity was sensitive to phenylmethylsulfonyl fluoride (PMSF), a serine protease inhibitor, indicating specific inhibition (data not shown). In contrast, CM prepared in the presence of PP2 exhibited reduced protease activities ( $>50 \%$ inhibition) (Figure 4), while PP3 exhibited no effects (Figure 4). The protease secretion was increasingly reduced with a corresponding increase in the concentration of PP2, an effect not observed when PP3 was used (Figure 4).

\section{Src kinases did not affect Acanthamoeba adhesion to and} cytotoxicity of HBMEC

To determine the involvement of Src kinase in A. castellanii binding to and cytotoxicity of HBMEC, assays were performed. It was observed that neither PP2 nor PP3 had any significant effect on Acanthamoeba binding to HBMEC monolayers (Figure 5A) (At $100 \mu \mathrm{M}, P=0.086$ for PP2 and $P=0.256$ for PP3 respectively, using $T$-test,

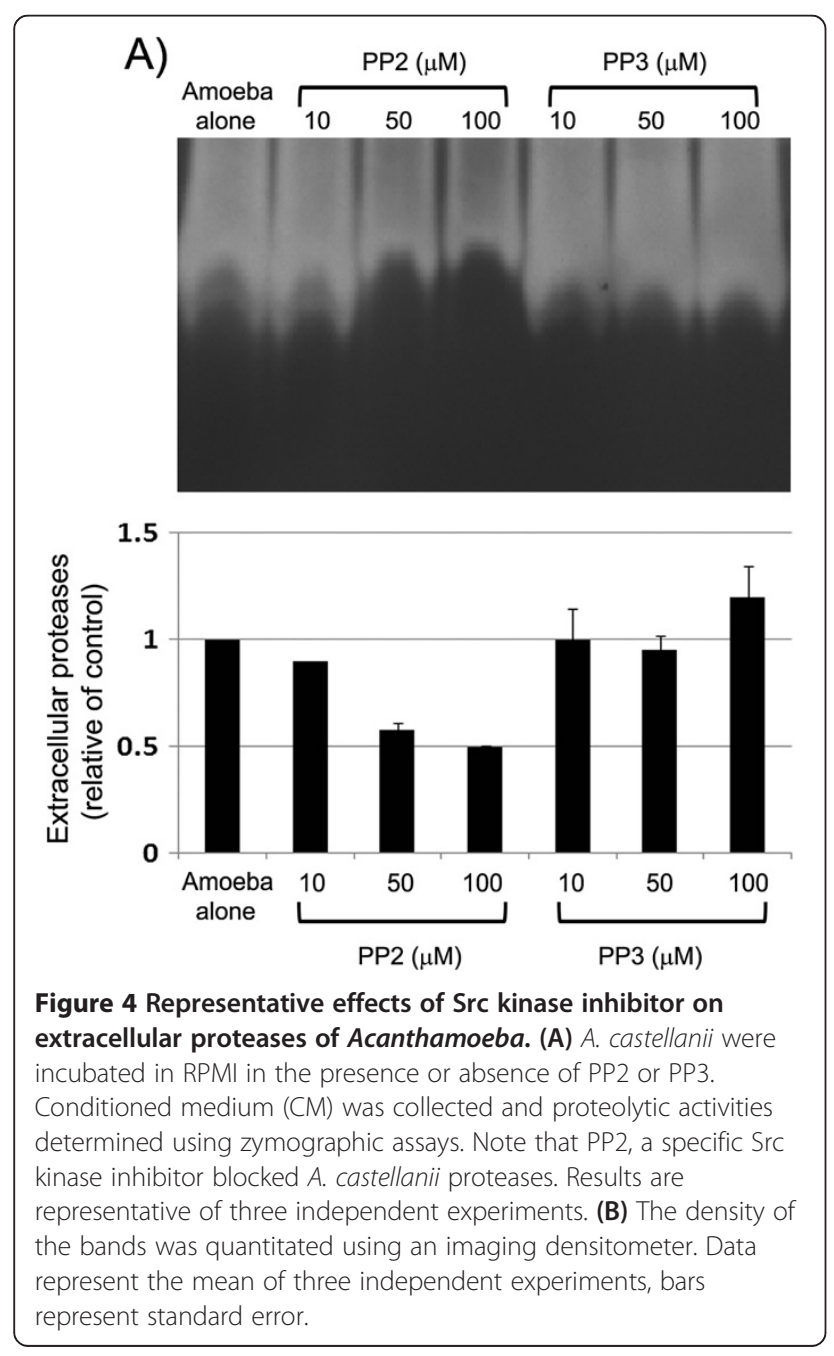

paired, one tail distribution). Next, to determine the effects of PP2 on A. castellanii-mediated HBMEC death, cytotoxicity assays were performed. In the absence of PP2, Acanthamoeba produced severe HBMEC cell cytotoxicity (up to 65\%) within $24 \mathrm{~h}$ (Figure 5B). Although A. castellanii pre-treatment with PP2 showed increased HBMEC cytotoxicity due to amoebae, however, these effects were insignificant (At $100 \mu \mathrm{M}$ of PP2, $P=0.053$, using $T$-test, paired, one tail distribution) (Figure $5 \mathrm{~B}$ ). In contrast, PP3 had no effects on $A$. castellanii-mediated HBMEC cytotoxicity (At $100 \mu \mathrm{M}$ of PP3, $P=0.10$, using $T$-test, paired, one tail distribution) (Figure $5 \mathrm{~B}$ ).

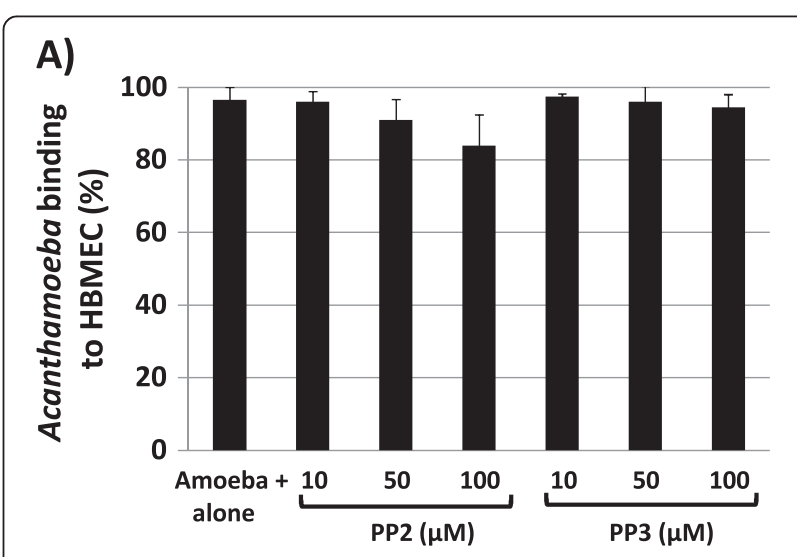

B)

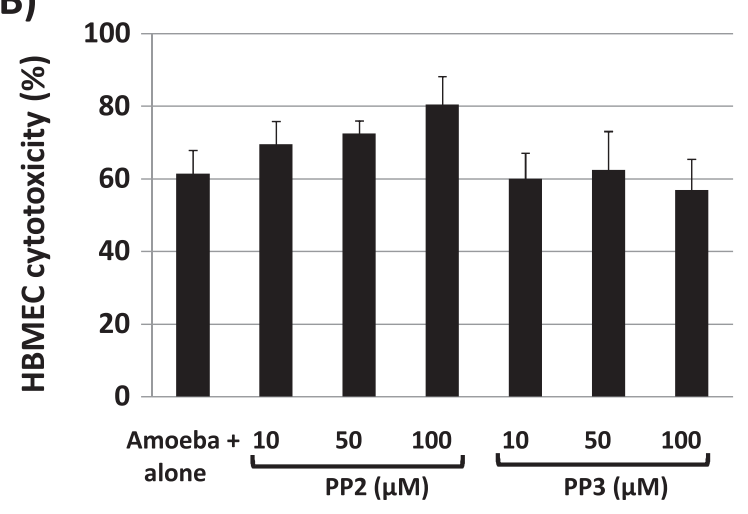

Figure 5 Inhibition of Src kinase had no effect on A. castellanii adhesion to and cytotoxicity of HBMEC. (A) Various concentrations of PP2 or PP3 were added to A. castellanii $\left(4 \times 10^{5}\right.$ amoebae/well) for $30 \mathrm{~min}$. After this incubation, A. castellanii were washed with PBS, and added to HBMEC monolayers for $1 \mathrm{~h}$. The unbound amoebae were counted using a haemocytometer and the numbers of bound amoebae were deduced as follows: No. of unbound amoebae/Total number of amoebae $\times 100=\%$ unbound amoebae. The numbers of bound amoebae were deduced as follows: \% unbound amoebae $-100=\%$ bound amoebae. (B) As for adhesion assays, amoebae were incubated with HBMEC for up to $24 \mathrm{~h}$ and the percent HBMEC cytotoxicity was determined by measuring $\mathrm{LDH}$ release. Results represent the mean of three independent experiments performed in duplicate. Bars represent standard error. 


\section{Acanthamoeba src kinase identification and sequence alignments}

A BLAST search of Monosiga brevicollis Src1 protein in the $A$. castellanii genome resulted in the identification of protein (of 639 amino acids in length), which was found to be similar in architecture to human $\mathrm{cSrc}$ and its orthologs (Figure 6a and b), indicated here as Acanthamoeba castellanii Src kinase (AcSrc). Putative AcSrc kinase shares considerable amino acid homology with its orthologs $[36 \%$ with Caenorhabditis elegans Src1 (NP_490866); 34\% with Ephydatia fluviatilis Src related protein (BAB83688); 33\% with Hydra vulgaris (AAA29217.1); 31\% with Drosophila melanogaster c-Src (AAA28913.1)]. However, it also has its unique $N$ - and $C$-terminal regions which are not homologous to its orthologs but to a small hypothetical protein of the fungus Verticillium dahliae in NCBI database (data not shown). In the human cSrc kinases protein, phosphorylation of tyrosine at positions 419 and 530 (Y419 and Y530) marks the protein for active and inactive state, respectively [21,22]. Of these two phosphorylation sites, a tyrosine residue corresponding to Y530 of human cSrc is present in AcSrc kinase, but the tyrosine residue corresponding to Y419 of human cSrc is absent. This information suggests that the AcSrc kinase might be constitutively active in Acanthamoeba. The role of these conserved tyrosine residue Y530 is not clear in protist Src kinases, because the phosphorylation of MbSrc1 in Monosiga brevicolli at the tyrosine residue corresponding to Y530 of human cSrc kinase did not result in an inactivation of its kinase activity, unlike its human homologs [20], which may explain the functioning of AcSrc. The amino acids which take part in binding of ATP and PP2 in human Srk kinases [23,24] showed similarity in AcSrc (Figure 6b). This further supported our findings that PP2 binds and inhibits Acanthamoeba Src kinase, which might have a role in important cellular processes, such as growth and phagocytosis.

Recent studies have shown that Acanthamoeba exhibits multifactorial virulence properties to produce damage of HBMEC, which constitute the blood-brain barrier, to produce encephalitis and corneal epithelial cells to induce keratitis [4]. Here, we studied the role of Src kinases on the biology and pathogenesis of Acanthamoeba. The findings revealed that Src kinase inhibitor, PP2, but not its inactive analog, PP3, abolished A. castellanii proliferation. These findings indicate that Src kinases have a pivotal role in the growth of A. castellanii, which is consistent with some previous findings that showed that Src is an important signalling molecule involved in the growth of various eukaryotic cell types $[11,12]$. Src kinases act on a number of substrates in the cell, including enzymes involved in phospholipid metabolism, such as the p85 subunit of PI3-K, phopholipase
C- $\gamma$ (PLC- $\gamma$ ) and the signalling molecules p190RhoGAP, p120rasGAP and EGF receptor substrate, Eps8, involved in cellular transformation, proliferation and survival [2527]. Phospholipid metabolism is important for the synthesis and maintenance of the cell membrane. Inhibition of this pathway would result in a cessation of growth and proliferation, a possible explanation for the present findings relating to the transformation of amoebae into the cyst form. The fact that Src kinases are involved in the life cycle and growth of A. castellanii suggest that they should be studied further.

A. castellanii use phagocytosis for food uptake (such as non-invasive E. coli $\mathrm{K}-12$ ), whereas $E$. coli $\mathrm{K} 1$ (a causative agent of bacterial meningitis), utilises A. castellanii as a reservoir and invades into the Acanthamoeba. Inhibition of Src kinase reduced A. castellanii phagocytosis of non-invasive $\mathrm{K}-12$ with an increase in the concentration of PP2, while opposite effects were observed for invasive $E$. coli $\mathrm{K} 1$. A likely explanation is that $E$. coli $\mathrm{K}-12$ is a food source for amoebae. In such situations, the process of bacterial uptake is most likely to be "driven" by A. castellanii, which would be dependent on amoeba intracellular signal transduction pathways involving Src kinases. In contrast, neuropathogenic E. coli $\mathrm{K} 1$ is an invasive strain. Their invasion of $A$. castellanii is most likely reliant on bacterial invasion, which might explain the involuntary uptake of $E$. coli $\mathrm{K} 1$ by $A$. castellanii, as observed in the present study. However, this contrasts with results of previous studies [16,28,29], which showed the involvement of Src in Staphylococcus aureus-invasion of embryonic kidney cells, Shigella invasion of epithelial cells and uropathogenic E. coli-invasion of bladder epithelial cells, which may be due to different host cell types, i.e., phagocytic Acanthamoeba versus non-phagocytic host cells. Future studies are needed to address these aspects in a comparative way.

Acanthamoeba are known to secrete large amounts of proteases that are shown to be key virulence factors in keratitis [30,31] as well as GAE infections [9,13]. For example, it is shown that serine proteases of Acanthamoeba increased the permeability of the blood-brain barrier by over $45 \%$ [32], while intrastromal injection of amoeba excretory products produced lesions similar to those observed in keratitis patients [31]. In the present study, it was observed that Src kinase played an important role in the protease secretion by $A$. castellanii. The proteases involved were shown to be serine proteases, inhibited by PMSF. This is significant as the inhibition of serine proteases dramatically reduce the ability of $A$. castellanii to cause inflammation and tissue damage $[13,32]$ and possibly subsequent GAE.

Previous studies have shown that the adhesion of $A$. castellanii to the host cell is an important step in the pathogenesis of acanthamoebiasis $[33,34]$. Our findings 


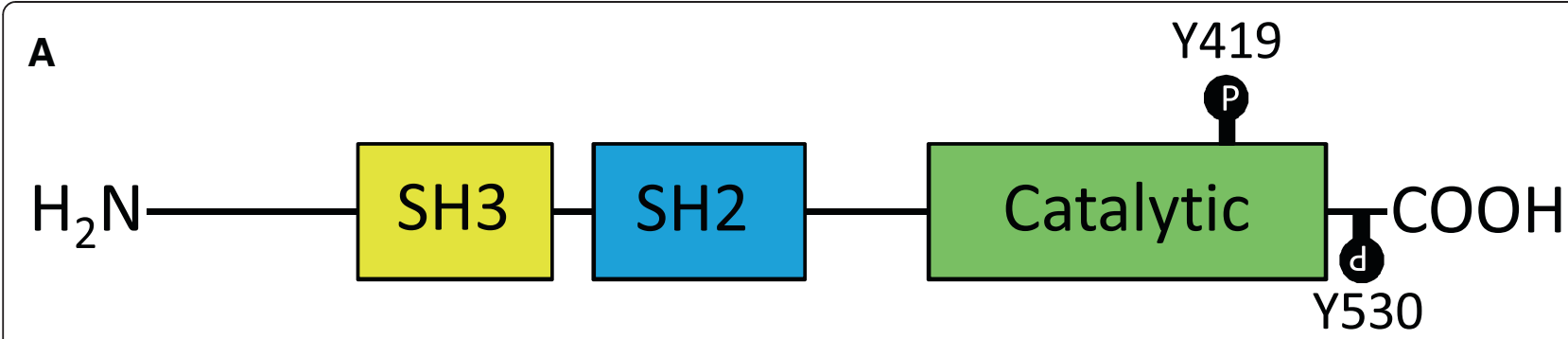

B

AcSrC

DmSrC

Cesrc

EfSrc

$\mathrm{MbSrc}$

Hvsrc

HsSrc

AcSrc

DmSrc

Cesrc

EfSrc

MbSrc

Hvsrc

HsSrc

AcSrc

DmSrc

Cesrc

EfSrc

MbSrc

Hvsre

HsSrc

AcSrc

DmSr

Cesrc

EfSrC

Mbsrc

Hvsre

HsSrC

AcSrc

DmSrc

Cesrc

EfSrC

MbSrc

Hvsrc

HsSrc

AcSrc

DmSrc

Cesrc

EfSrc

MbSrc

Hvsrc

HsSrC

625

GAGDDSSVFSPYGSVELRRGAESSADDTSCDEAEAVEMEREAKQANDKK

1

250

275 375 SFS- -

$600-2$

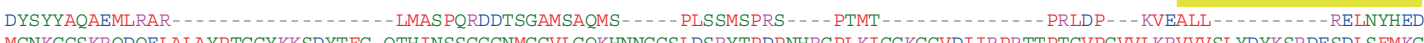
MGNKCCSKRQDQELALAYPTGGYKKSDYTFG-QTHINSSGGGNMGGVLGQKHNNGGSLDSRYTPDPNHRGPLKIGGKGGVDI IRPRTTPTGVPGVVLKRVVVSLYDYKSRDESDLSFMKG MGCLFSKERRSGGSDMGVSERTDVS - - - RFOTPOQOTVFHVNNGGNE- - - n - GTIS - - - OLNGTSDGMMGNGRG - - GGGGGGAOERETLVALYPYDSRADGDISFOKC

FADTPQKANDVKIEFSQSQNSVQLPTARVATPP - - - PLPO

MGPCCSKQTKA

PV- PGOVPODDDPNLFIALYDYEARTADDLTFQKG

-MHVSHNQPPNINPP - - -MLGGP- - -GVTIFVALYDYEARISEDLSFKKG -KLFGGFNSDTVTSP-- QRAGPLAGGVTTFVALYDYESRTETDLSFKKG

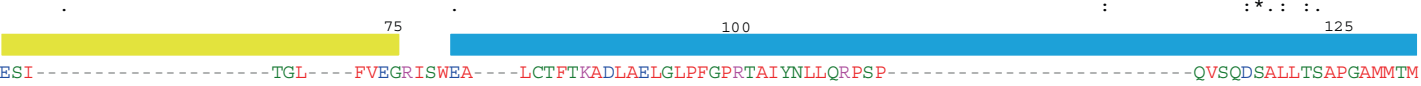
DRMEVIDDTESDWWRVVNLTTROEGLI PLNFVAEERSVNSEDWFFENVLRKEADKLLLAEENPRGTFLVRPSEHNPNGYSLSVKDWEDGR -GYHVKHYRI KPLD - NGGYYIATNOTFPSI DAMYLIDHSNCDWWYVRHQRTGQTGYVPRNFVAKQQTIESEEWYAGKIPRNRAERLVLSSHLPKGTFLIREREADTREFALTIRDTDDQRNGGTVKHYKI KRLDHDQGYF ITTRRTFRSL DKLI I LAGLDGDWWMARSTKTNKEGYI PRNYVAPLASYEAEDWYFGDVGRAEAEKWLLAPGTQSGAFLVRASSTQKNSLSLSLRD- - - - GEG IKHYRIRTLD - NGGFF IANRISFPTL EKLKI TNNSDGDWWQATSLITGKSGYI PSNFVAAVQS IESEDWYHGRIKRAEAEKVLMLTGVE-GSFLIRESEKP-GQYALS IRT- - - - GDIVKHYSIRTLD - EGGYYITSRVTFRTL FRLOI TNTADGDWWYARSLITNSEGYT PSTYVAPEKSYEAEEWYFGDVKRAEAEKRLMVRGL PSGTEL TRKAETAVGNFSLSVRD - GDSVKHYVRKID TGGYFTTTRADFNST ERLQIVNNTEGDWWLAHSLSTGQTGYI PSNYVAPSDS IQAEEWYFGKITRRESERLLLNAENPRGTFLVRESETTKGAYCLSVSDFDNAK-GLNVKHYKIRKLD-SGGFYITSRTOFNSL

\section{: $\quad$ * $\quad:$ *}

$\begin{array}{cccc}150 & 175 & 200 & 225 \\ \text { M- } & & 175\end{array}$ QALVMAYSKNALGLCHILSRPC- PKPQPQMWDLGPELRDKYEI PRSEIQLLRKLGRGNFGEVFYGKWRNS IDVAVKTLREGTMSTAAFLQEAAIMKKFR- OELVRYYSDVPDGLCCOLTFPA- PRLAPTRPDLSHDTOONWEI PRNQLHLKRKLGDGNFGEVWYGKWRGIVEVAIKTMKPGTMS PEAFLOEAOIMKOCD - QDLVAHYMMDSDGLAAQRLAMPCSRANI PITSGLS- -YKDEWEIDRSTIVLORKLGOGNFGEVWAGVWNGTTAVAVKTLKPDTMEVKDFVOEAOVMKKIH- OELVSHYRESADGLTCKLREPCRPISOPETVGLDVHTKDOWEI PRKS TELKSKLGSGOFGDVWRGVWNKTTEVAVKTLKPGSMSAFEFLKEAGVMKRLRYELVQHYTKDADGLVCALTLPC - PKDKPVTGGI - - AKDAWEI PRESLRLNRKLGAGQFGEVWAGVWNNTTQVAVKTLKPGTMS PASFLDEAGVMKKLR - QQLVAYYSKHADGLCHRLTTVC - PTSKPQTQGL - - AKDAWEI PRESLRLEVKLGQGCFGEVWMGTWNGTTRVAIKTLKPGTMS PEAFLQEAQVMKKLP $*^{* *} * *^{* *}: * *$. ${ }^{*} * *^{*}:$.

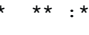

PHDTHANVLCIVMCDVCSELPAHANVVTFYGVADADKGVYIVTEFVPDGSLDRYLM-EORDVIEOSVLVOMI KDVAAGMDHLATHG ILHRDLAARNLLVEVKRSGYHRVKVCDFGLALER HNRLVALYAVCSQEEPIYIVQEYMSKGSLIDFLREGDGRYLHFEDLIYIATOVASGMEYLESKQLIHRDLAARNVLIGENN - - - -VAKICDFGLA- HPNLVKL YAVCTRE -HPNLLQLYAVCTIGEPIYIVTELMKFGSLLEYLKHGEGKNITLHQMIDMSAQ IASGMTYLEAHSYIHRDLAARN ILVGEGN - - - -VCKVADFGLA - - -HPKLIQLYAVCTDKEPIYIVTELMKNGSLLDYLH-DKGRALNLPQLVDMAAQVASGMAYLEAQNFVHRDLAARNVLVGDNN - - - -TCKVGDFGLS - - - - - -HKHLVQLYAICSDREPIYIVTEYMSGGSLIDYLSKGEGVNLQLPTLIDMAAQVASGMAFLEAQGYIHRDLAARNILVGENY - - - ICKVADFGLA - - - 法

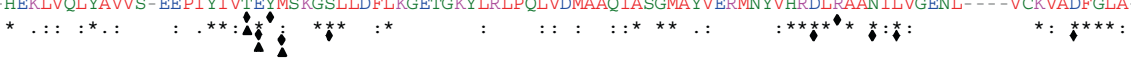
425 450

AASDGPVTDEQLLQOEQOQRLSNVPVRWTAI EVLLHGHYTTKSDVWSFGVTVWEI FSGGQIPYADLSTFEIVELLLGGYKLPRPERC- - PEAVYERIILRCLADDPDDRPTFHEIYRTMD -RVI - ADDEYCPKQGSRF - - PVKWTAP A I IYGKFS IKSDVWSYGIL - RVI-ADDEYCPKQGSRF - - -PVKWTAPEAIIYGKF SIKSDVWSYGILLMELFTYGQVPYPGMHSREVIENIERGFRMPKPTNHYFPDNIY-QLLLLCWDAVPEKRPTFEFLNHYFE

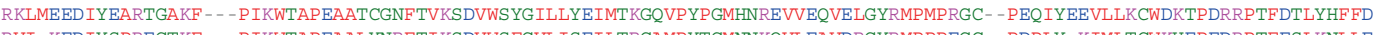
-RVI-KEDIYSPREGTKF - - PIKWTAPEAAL YNRFTI KSDVWSFGVLISEI LTRGAMPYTGMNNKQVLEAVDRGYRMP PPEGC - PDPLY -KIMLTCWKHEPEDRPTFESLKNLLE -RVLGQESEYTAREGAKF - - PIKWTAPEAALMNRF S IKSDVWAFG ILLTELVTYGR IPYPGMTNAEVLQQVERGYRMPAPTNC - PPELY -N IMLDCWKFKPDERPTFETLQYRLE -RLI -EDDEYTAHEGAKF - - PIKWTAPEAALYNRFTI KSDVWSFGILMAEIVTKGR IPYPGMTNAQTIAEVEKGYRMP IMPGC - PEPLY -NIMLQTWNKDPENRPTFDYLQGVLE
475
500
525
550
575

ALFPTIYASAVIDLSPAGTGGLRRSAPSAQAQRKPSISSFVPRLAFASTPNLASAAAAPSSLLVSSSSVPTSAGSSPRYNASTSSSSVFSPYSS IAMPEASSDDSDSESDSESESEDDIC -

DYF- -

DYY -

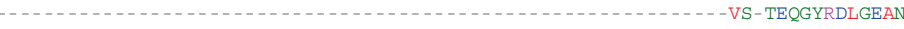

¿

AcSrc

Dmsrc

DmSrC

Cesrc

$\operatorname{MbSrC}$

Hvsre

HsSrc

Figure 6 (See legend on next page.) 
(See figure on previous page.)

Figure 6 Sequence alignment of Acanthamoeba castellanii Src kinase and its orthologs. (a) Domain architecture of human c-Src kinase. The two important regulatory phosphorylation sites (Y419 and Y530) are marked. (b) Putative Acanthamoeba Src kinase (AcSrc) sequence was aligned with Drosophila melanogaster c-Src (DmSrc), Caenorhabditis elegans Src (CeSrc), Ephydatia fluviatilis Src related protein (EfSrc), Monosiga brevicollis $\mathrm{Src1}(\mathrm{MbSrc})$, Hydra vulgaris (HvSrc) and Homo sapiens $\mathrm{CSrc}(\mathrm{HsSrc})$. The SH3, SH2 and catalytic domain are indicated with yellow, blue and green bars above the sequence, respectively. Asterisk (*), colon (:) and period (.) marks the region of conserved, strongly similar and weakly similar residues, respectively. Whereas black diamond represents residues involve in ATP binding; black arrowheads represents residues form hydrogen bond with PP2, and black circles indicates the Y419 and Y530 phosphorylation sites in human cSrc, most of which are also conserved in AcSrc.

revealed that Src kinase had a minimal effect on $A$. castellanii adhesion to HBMEC, which is not surprising, as Src kinases are regulators of intracellular signalling pathways, and the binding of amoebae to HBMEC is known to be mediated by mannose-binding protein expressed on the surface membranes of Acanthamoeba [33,34]. Given that Src kinases inhibited protease secretion and phagocytic activity of $A$. castellanii and that both properties have been implicated in the pathogenicity of Acanthamoeba, it was anticipated that inhibition of Src kinases would result in reduced A. castellanii-mediated HBMEC cytotoxicity. It was surprising that the inhibition of Src kinases had no significant effect on HBMEC cytotoxicity. However, cytotoxicity is a delayed event and PP2 effects are reversible [19]. The fact that assays were performed by pre-treatment of $A$. castellanii with PP2 for $30 \mathrm{~min}$ followed by their incubation with HBMEC for $24 \mathrm{~h}$. in the absence of inhibitor, may explain these findings. As PP2 has toxic effects on the host cells, assays could not be performed in the presence of PP2 for longer periods of time. Future studies, using host cells expressing dominant negative forms of Src kinases, should establish their involvement in A. castellaniimediated HBMEC cytotoxicity.

\section{Conclusions}

For the first time, the present findings showed that Src kinases are involved in the proliferation, phagocytic properties and proteolytic activities of $A$. castellanii. Future studies should further explore the precise mechanisms associated with Acanthamoeba pathogenesis, which may help develop preventative and/or therapeutic interventions against acanthamoebiasis.

\section{Competing interests}

The authors declare that they have no competing interests.

\section{Acknowledgements}

This work was partially supported by grants from The Aga Khan University, Life Sciences Research Fund, University of London, and British Council for Prevention of Blindness.

\section{Author details}

'Department of Biological and Biomedical Sciences, The Aga Khan University, Karachi, Pakistan. ${ }^{2}$ School of Biological and Chemical Sciences, University of London, Birkbeck, UK.

\section{Authors' contributions}

NK conceived the study. RS and MM designed and conducted all experiments under the supervision of NAK. Jl performed genome analyses and interpretations. RS, Jl and NAK contributed to the writing of the manuscript. All authors approved the final manuscript.

Received: 2 April 2012 Accepted: 15 May 2012

Published: 7 June 2012

\section{References}

1. Booton GC, Visvesvara GS, Byers TJ, Kelly DJ, Fuerst PA: Identification and distribution of Acanthamoeba species genotypes associated with nonkeratitis infections. J Clin Microbiol 2005, 43:1689-1693.

2. Corsaro D, Venditti D: Phylogenetic evidence for a new genotype of Acanthamoeba (Amoebozoa, Acanthamoebida). Parasitol Res 2010, 107:233-238.

3. Nuprasert W, Putaporntip C, Pariyakanok L, Jongwutiwes S: Identification of a novel 117 genotype of Acanthamoeba from environmental isolates and t10 genotype causing keratitis in Thailand. I Clin Microbiol 2010, 48:4636-4640.

4. Siddiqui R, Khan NA: Biology and Pathogenesis of Acanthamoeba. Parasit Vectors 2012, 5:6

5. Marciano-Cabral F, Cabral G: Acanthamoeba spp. as agents of disease in humans. Clin Microbiol Rev 2003, 16:273-307.

6. Visvesvara GS, Moura H, Schuster FL: Pathogenic and opportunistic freeliving amoebae: Acanthamoeba spp., Balamuthia mandrillaris, Naegleria fowleri, and Sappinia diploidea. FEMS Immunol Med Microbiol 2007, 50:1-26.

7. Croft SL, Seifert K, Duchêne M: Antiprotozoal activities of phospholipid analogues. Mol Biochem Parasitol 2003, 126:165-172.

8. Martinez AJ, Visvesvara GS: Free-living, amphizoic and opportunistic amebas. Brain Pathol 1997, 7:583-598.

9. Khan NA: Acanthamoeba and the blood-brain barrier: the breakthrough J Med Microbiol 2008, 57:1051-1057.

10. Chiarugi P: Src redox regulation: there is more than meets the eye. Mol. Cell 2008, 26:329-337.

11. Roskoski R: Src protein-tyrosine kinase structure and regulation. Biochem Biophys Res Commun 2004, 324:1155-1164.

12. Tatosyan AG, Mizenina OA: Kinases of the Src Family: structure and functions. Biochem 2000, 65:49-58.

13. Khan NA, Siddiqui R: Acanthamoeba affects the integrity of the human brain microvascular endothelial cells and degrades the tight junction proteins. Int J Parasitol 2009, 39:1611-1616.

14. Stins MF, Gilles F, Kim KS: Selective expression of adhesion molecules on human brain microvascular endothelial cells. J Neuroimmunol 1997, 76:81-90.

15. Hanke JH, Gardner JP, Dow RL, Changelian PS, Brissette WH, Weringer EJ, Pollok BA, Connelly PA: Discovery of a novel, potent, and Src familyselective tyrosine kinase inhibitor. Study of Lck- and FynT-dependent T cell activation. J Biol Chem 1996, 271:695-701.

16. Eto DS, Jones TA, Sundsbak JL, Mulvey MA: Integrin-mediated host cell invasion by type 1-piliated uropathogenic Escherichia coli. PLoS Pathog 2007, 3:e100.

17. Sissons J, Alsam S, Stins M, Rivas AO, Morales UL, Faull J, Khan NA: Use of in vitro assays to determine effects of human serum on biological characteristics of Acanthamoeba castellanii. J Clin Microbiol 2006, 44:2595-2600. 
18. Matin A, Stins M, Kim KS, Khan NA: Balamuthia mandrillaris exhibits metalloprotease activities. FEMS Immunol Med Microbiol 2006, 47:83-91.

19. Sissons J, Kim KS, Stins M, Jayasekera S, Alsam S, Khan NA: Acanthamoeba castellanii induces host cell death via a phosphatidylinositol 3-kinase-dependent mechanism. Infect Immun 2005, 73:2704-2708.

20. Barker SC, Kassel DB, Weigl D, Huang X, Luther MA, Knight WB: Characterization of pp 60c-src tyrosine kinase activities using a continuous assay: autoactivation of the enzyme is an intermolecular autophosphorylation process. Biochem 1995, 34:14843-14851.

21. Boggon TJ, Eck MJ: Structure and regulation of Src family kinases. Oncogene 2004, 23:7918-7927.

22. Li W, Young SL, King N, Miller WT: Signaling properties of a non-metazoan Src kinase and the evolutionary history of Src negative regulation. J Biol Chem 2008, 283:15491-15501.

23. Sicheri F, Kuriyan J: Structures of Src-family tyrosine kinases. Curr Opin Struct Biol 1997, 7:777-785.

24. Zhu X, Kim JL, Newcomb JR, Rose PE, Stover DR, Toledo LM, Zhao H, Morgenstern KA: Structural analysis of the lymphocyte-specific kinase Lck in complex with non-selective and Src family selective kinase inhibitors. Structure 1999, 7:651-661.

25. Kassenbrock CK, Hunter S, Garl P, Johnson GL, Anderson SM: Inhibition of Src family kinases blocks epidermal growth factor (EGF)-induced activation of Akt, phosphorylation of c-Cbl, and ubiquitination of the EGF receptor. J Biol Chem 2002, 277:24967-24975.

26. Jin W, Yun C, Jeong J, Park Y, Lee HD, Kim SJ: C-Src is required for tropomyosin receptor kinase $\mathrm{C}$ (TrkC)-induced activation of the phosphatidylinositol 3-kinase (PI3K)-AKT pathway. J Biol Chem 2008, 283:1391-1400.

27. Windham TC, Parikh NU, Siwak DR, Summy JM, McConkey DJ, Kraker AJ, Gallick GE: Src activation regulates anoikis in human colon tumor cell lines. Oncogene 2002, 21:7797-7807.

28. Agerer F, Michel A, Ohlsen K, Hauck CR: Integrin-mediated invasion of Staphylococcus aureus into human cells requires Src family proteintyrosine kinases. J Biol Chem 2003, 278:42524-42531.

29. Mounier J, Popoff MR, Enninga J, Frame MC, Sansonetti PJ, Van Nhieu GT: The IpaC carboxyterminal effector domain mediates Src-dependent actin polymerization during Shigella invasion of epithelial cells. PLoS Pathog 2009, 5:e1000271.

30. Cao Z, Jefferson DM, Panjwani N: Role of carbohydrate-mediated adherence in cytopathogenic mechanisms of Acanthamoeba. J Biol Chem 1998, 273:15838-15845.

31. He YG, Niederkorn JY, McCulley JP, Stewart GL, Meyer DR, Silvany R, Dougherty J: In vivo and in vitro collagenolytic activity of Acanthamoeba castellanii. Invest Ophthalmol Vis Sci 1990, 31:2235-2240.

32. Alsam S, Sissons J, Jayasekera $S$, Khan NA: Extracellular proteases of Acanthamoeba castellanii (encephalitis isolate belonging to $\mathrm{T} 1$ genotype) contribute to increased permeability in an in vitro model of the human blood-brain barrier. J Infect 2005, 51:150-156.

33. Alsam S, Kim KS, Stins M, Rivas AO, Sissons J, Khan NA: Acanthamoeba interactions with human brain microvascular endothelial cells. Microb Pathogen 2003, 35:235-241

34. Garate M, Cao Z, Bateman E, Panjwani N: Cloning and characterization of a novel mannose-binding protein of Acanthamoeba. J Biol Chem 2004 279:29849-29856.

\section{Submit your next manuscript to BioMed Central and take full advantage of:}

- Convenient online submission

- Thorough peer review

- No space constraints or color figure charges

- Immediate publication on acceptance

- Inclusion in PubMed, CAS, Scopus and Google Scholar

- Research which is freely available for redistribution 